\title{
Pattern analysis approach reveals restriction enzyme cutting abnormalities and other CDNA library construction artifacts using raw EST data
}

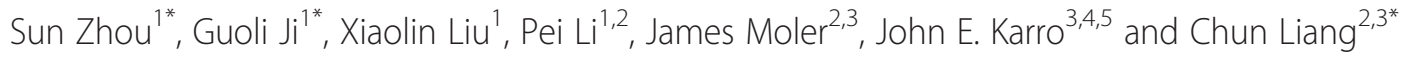

\begin{abstract}
Background: Expressed Sequence Tag (EST) sequences are widely used in applications such as genome annotation, gēne discovery and gēne expression studies. However, some of GenBank dbEST sequences have proven to be "unclean". Identification of cDNA termini/ends and their structures in raw ESTs not only facilitates data quality control and accurate delineation of transcription ends, but also furthers our understanding of the potential sources of data abnormalities/errors present in the wet-lab procedures for cDNA library construction.

Results: After analyzing a total of 309,976 raw Pinus taeda ESTs, we uncovered many distinct variations of cDNA termini, some of which prove to be good indicators of wet-lab artifacts, and characterized each raw EST by its CDNA terminus structure patterns. In contrast to the expected patterns, many ESTs displayed complex and/or abnormal patterns that represent potential wet-lab errors such as: a failure of one or both of the restriction enzymes to cut the plasmid vector; a failure of the restriction enzymes to cut the vector at the correct positions; the insertion of two cDNA inserts into a single vector; the insertion of multiple and/or concatenated adapters/linkers; the presence of $3^{\prime}$-end terminal structures in designated 5 '-end sequences or vice versa; and so on. With a close examination of these artifacts, many problematic ESTs that have been deposited into public databases by conventional bioinformatics pipelines or tools could be cleaned or filtered by our methodology. We developed a software tool for Abnormality Filtering and Sequence Trimming for ESTs (AFST, http://code.google.com/p/afst/) using a pattern analysis approach. To compare AFST with other pipelines that submitted ESTs into dbEST, we reprocessed 230,783 Pinus taeda and 38,709 Arachis hypogaea GenBank ESTs. We found 7.4\% of Pinus taeda and 29.2\% of Arachis hypogaea GenBank ESTs are "unclean" or abnormal, all of which could be cleaned or filtered by AFST.

Conclusions: cDNA terminal pattern analysis, as implemented in the AFST software tool, can be utilized to reveal wet-lab errors such as restriction enzyme cutting abnormities and chimeric EST sequences, detect various data abnormalities embedded in existing Sanger EST datasets, improve the accuracy of identifying and extracting bona fide cDNA inserts from raw ESTs, and therefore greatly benefit downstream EST-based applications.
\end{abstract}

Keywords: cDNA terminus, cDNA library construction, Pattern analysis, Restriction enzyme cutting abnormality, Chimeric EST sequences

\footnotetext{
*Correspondence: zhousun@xmu.edu.cn; glji@xmu.edu.cn; liangc@muohio.

edu

${ }^{1}$ Department of Automation, Xiamen University, Xiamen, Fujian 361005, China

2Department of Botany, Oxford, OH 45056, USA

Full list of author information is available at the end of the article
} 


\section{Background}

ESTs are primarily cDNA sequences obtained by sequencing cDNA fragments/clones made from mRNAs. Representing transcribed portions of various genomes, ESTs are widely used for a variety of genomic researches, including novel gene discovery, gene expression studies, and genome annotation [1-8]. While cDNA/EST data generated by next-generation sequencing technologies (such as 454 or Illumina) is being deposited into the NCBI Short Read Archive (http://www.ncbi.nlm.nih.gov/Traces/sra/sra.cgi) in an unprecedented rate, the quantity of publically available EST data created by traditional Sanger sequencing is still increasing. As of November 1, 2011, there were $71,235,293$ entries deposited in the GenBank dbEST, the public data repository for traditional Sanger ESTs [9]. Unfortunately, many EST datasets are poorly processed, and GenBank dbEST contains numerous errors from a range of sources. For example, double-termini adapters, the palindrome linker sequences that likely concatenate two different transcripts to form chimeric ESTs, were identified in many Pinus teada ESTs [10]. In another case, we were able to identify a number of spurious sequence remnants (i.e. vector or adapter fragments) in a large portion of the GenBank ESTs and their clusters/contigs for Chlamydomonas reinhardtii [11], an artifact of undertrimming during the procedures of raw EST cleanup.

In order to significantly reduce the errors in public EST databases, we proposed a protocol that processes raw EST data based on cDNA termini/ends - a set of diagnostic sequence elements that can be used to delineate cDNA insert ends and facilitate extraction of bona fide cDNA insert sequences from raw ESTs [11,12]. Specifically, the diagnostic sequence elements for cDNA termini include adapter/linker sequences, insert-flanking restriction enzyme recognition sites, poly (A)/(T) tails, and plasmid vector fragments immediately adjacent to cDNA inserts. Moreover, these individual elements or components must have retained their sequential order and orientation constraints and form a canonical or expected structure for a given cDNA terminus, known as the cDNA terminus structure [11]. Our previous work [11,12] focused on detecting canonical cDNA terminal structures expected from the adopted cDNA library constructional protocols and filtering out those ESTs with abnormal and complex terminal structures for downstream applications. In this study, we have collected a total of 309,976 raw Pinus taeda EST trace files, the majority of which have been submitted to both NCBI dbEST and Trace Archive. Using this dataset, our objective is to characterize the abnormal and complex terminus structure patterns, explore the potential underlying sources of wet-lab artifacts/errors, and develop a new EST cleaning software tool based on pattern analysis approach. Using our new tool, we have reprocessed 230,783 Pinus taeda and
38,709 Arachis hypogaea GenBank ESTs, and detected a significant number of problematic EST sequences. Clearly, characterization of abnormal and complex terminal structures will improve current EST cleaning steps and facilitate the quality control of error-prone ESTs.

\section{Results and Discussion}

\section{Pattern analysis of abnormal cDNA terminal structures}

In our previous studies [11,12], we defined four canonical cDNA termini: $\underline{\mathbf{5}}$ terminus of the cDNA in the sense

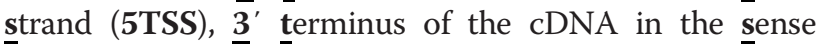

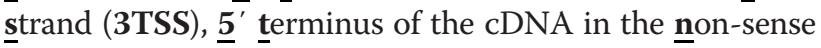
(anti-sense) strand (5TNS), and $\underline{\mathbf{3}}^{\prime}$ terminus of the cDNA in the non-sense strand (3TNS). In particular, 5TSS and 3TSS denote the $\overline{5}^{\prime}$ and $3^{\prime}$ ends of the relevant mRNA, respectively, in the sense strand, whereas 5TNS and 3TNS delineate the $3^{\prime}$ and $5^{\prime}$ ends of an mRNA, respectively, and whose sequences are read in the $5^{\prime} \rightarrow 3^{\prime}$ direction in the non-sense strand. In order to better characterize the abnormal and complex terminus structures, in this study we have expanded our cDNA terminus definitions by adding more sub-components, as shown in Figure 1. For example, 3TSS-1 represents the combination of a poly(A) tail and a XhoI site (CTCGAG, Enzyme2); 3TSS-2 denotes the combination of a XhoI site (CTCGAG, Enzyme2) and the adjacent plasmid vector fragment marked as Vector fragment 2 (VF2); 3TSS-3 represents the poly(A) tail; 3TSS-4 denotes direct adjunction of a poly(A) tail, a guanine (G) instead of a XhoI site (CTCGAG, Enzyme2), and the vector fragment $V F 2$, which is impossible in theory; and 3TSS-5 stands only for the vector fragment $V F 2$. In Figure $1, V F 1$ and $V F 2$ are referred to the left and right vector borders of the cloning sites.

Using the same or similar cDNA library construction protocol illustrated in Figure 1, 309,976 raw ESTs for Pinus teada were generated by three different labs UGALAB $(172,229)$, NCSUFBG $(75,001)$ and TIGR_JCVIJTC (62,746). Among the UGALAB ESTs, we found that $82 \%(141,914$ out of 172,229$)$ contain detectable cDNA termini. Of those, about 38\% (54,112 out of $141,914)$ match the expected terminal structures described in Figure 1, while $62 \%(87,802$ out of 141,914$)$ possessed abnormal terminal structures that were different from the expected structures. In contrast, among the ESTs from NCSUFBG and TIGR_JCVIJTC, 94\% (70,589 out of $75,001)$ and $99 \%(62,253$ out of 62,746$)$ have detectable cDNA termini respectively. Of the identified ESTs, NCSUFBG has $68 \%(47,845$ out of 70,589$)$ with the expected cDNA terminal structures and 32\% (22,744 out of 70,589) with abnormal terminal structures, whereas TIGR_JCVIJTC has 44\% $(27,368$ out of 62,253$)$ with expected cDNA terminal structures and 56\% (34,885 out 


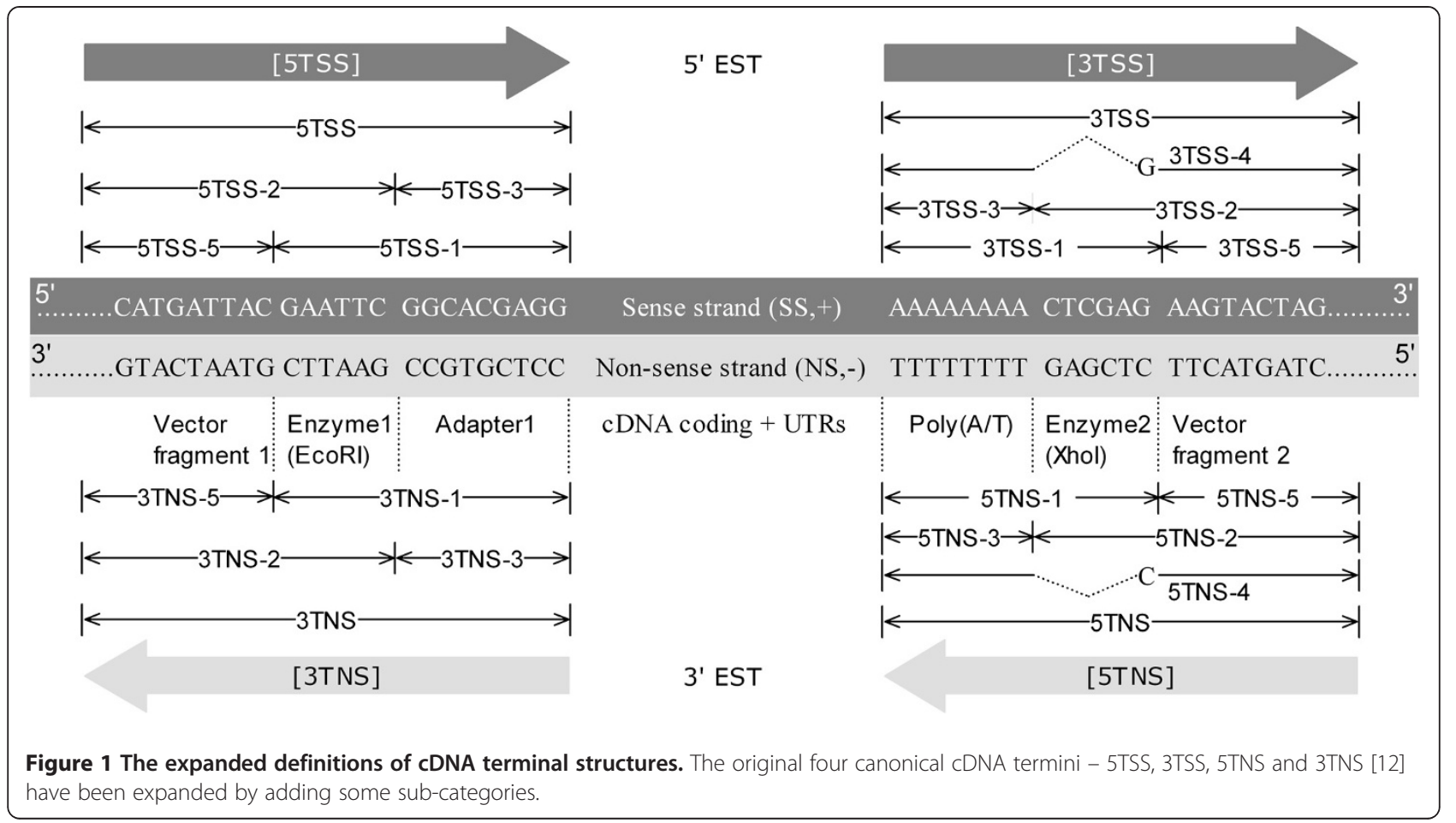

of 62,253) with abnormal terminal structures. Clearly, ESTs from UGALGB display more complex cDNA terminal patterns than the other two data sources.
Table 1 lists the most frequent abnormal patterns of cDNA termini detected in the 172,229 UGALAB ESTs generated using the exact protocol shown in Figure 1.

Table 1 The top patterns of abnormal sequences detected in 172, 229 Pinus taeda ESTs generated from UGALGB

\begin{tabular}{|c|c|c|c|c|c|c|}
\hline Case No. & EST Direction & Terminal Pattern ${ }^{1}$ & Number $^{2}$ & $\%^{3}$ & Description & Example $^{4}$ \\
\hline 1 & $5^{\prime}$ & $\mathrm{N}, 3 \mathrm{TSS}-3, \mathrm{~N}, \ldots, 3 \mathrm{TSS}-3, \mathrm{~N}$ & 27426 & $30.74 \%$ & $\begin{array}{l}\text { Single or multiple } \\
\text { poly(A) fragment(s). }\end{array}$ & Additional file 1: Figure S1 A \\
\hline 2 & $3^{\prime}$ & $(\mathrm{N}, 5 \mathrm{TNS}-4, \mathrm{~N}$ & 7707 & $9.28 \%$ & $\begin{array}{l}\text { Xhol site (CTCGAG) is } \\
\text { replaced by C. }\end{array}$ & Additional file 1: Figure S1 B \\
\hline 3 & $3^{\prime}$ & $(\mathrm{N}, 5 \mathrm{TNS}-5, \mathrm{~N}$ & 6814 & $8.21 \%$ & $\begin{array}{l}\text { The vector fragment } 2 \text { (VF2) } \\
\text { hasbeen identified, without } \\
\text { adjacent poly(T) tail and Xhol } \\
\text { site detected. }\end{array}$ & Additional file 1: Figure S1 D \\
\hline 4 & $5^{\prime}$ & N,3TSS-5,V & 1414 & $1.59 \%$ & $\begin{array}{l}\text { The vector fragment } 2 \text { (VF2) } \\
\text { hasbeen identified, without } \\
\text { poly(A) tail and Xhol detected. }\end{array}$ & Additional file 1: Figure S1 E \\
\hline 5 & $5^{\prime}$ & $\mathrm{N}, 5 \mathrm{TNS}-1, \mathrm{~N}$ & 1386 & $1.55 \%$ & $\begin{array}{l}\text { 3'-like terminus, i.e., poly(T) } \\
\text { and Xhol site, without the } \\
\text { vectorfragment 2 (VF2) } \\
\text { detected. }\end{array}$ & Additional file 1: Figure $\mathrm{S} 1 \mathrm{H}$ \\
\hline 6 & $3^{\prime}$ & 5TNS-2,N & 1238 & $1.49 \%$ & $\begin{array}{l}\text { Xhol site and the vector } \\
\text { fragment } 2 \text { (VF2) identified, } \\
\text { without a poly(T) tail detected. }\end{array}$ & Additional file 1: Figure S1 F \\
\hline 7 & $5^{\prime}$ & $\mathrm{N}, 3 \mathrm{TSS}-5, \mathrm{~N}$ & 873 & $0.98 \%$ & $\begin{array}{l}\text { The vector fragment } 2 \text { (VF2) } \\
\text { hasbeen identified, without } \\
\text { adjacent poly(A) tail and Xhol } \\
\text { site detected }\end{array}$ & Additional file 1: Figure S1 G \\
\hline 8 & $5^{\prime}$ & N,3TSS-4,V & 800 & $0.90 \%$ & Xhol site (CTCGAG) is replaced by G. & Additional file 1: Figure S1 C \\
\hline
\end{tabular}

${ }^{1} V$ stands for vector sequence while $N$ stands for non-vector sequence.

2 Total sequence numbers for a given case

${ }^{3}$ Of 172,229 ESTs, 83,021 are designated as 3'-ESTs (with ".b" in their sequence names) whereas 89,208 as 5'-ESTs (".g" in their sequence names). The percentage is calculated using the total sequence number for each case divided by all 3'-end or 5'-end ESTs.

${ }^{4}$ All examples are displayed in Additional file 1: Figure S1. 
The most frequent pattern (Case No.1, making up $30.74 \%$ of all $5^{\prime}$-end ESTs) is a single or multiple poly(A) fragments identified near the end of 5 '-EST sequences without either a flanking XhoI enzyme site or the vector fragment VF2 in proximity (Additional file 1: Figure S1 A). The second most frequent pattern (Case No. 2, 9.28\%) is the replacement of a XhoI site (CTCGAG, Enzyme2) by a single Cytosine (C) that is flanked by the vector fragment VF2 in the front and by the poly(T) tail at the end in 3'-end ESTs (see 5TNS-4 in Figure 1 and Additional file 1: Figure S1 B). The counterpart pattern of Case No. 2 in $5^{\prime}$-end ESTs is Case No. 8 (0.90\%) where a XhoI site (CTCGAG) is replaced by a single Guanine (G) that is flanked by the poly(A) tail in the front and by the vector fragment VF2 at the end in the sense strand of cDNA (see 3TSS-4 in Figure 1 and Additional file 1: Figure S1 C). The cDNA terminal pattern Cases No. 3 (8.21\%, Additional file 1: Figure S1 D), No. 4 (1.59\%, Additional file 1: Figure S1 E), No. 6 (1.49\%, Additional file 1: Figure S1 F) and No. 7 (0.98\%, Additional file 1: Figure S1 G) reflect the common theme: cDNA inserts are not flanked by a poly $(\mathrm{A}) / \operatorname{poly}(\mathrm{T})$ tail near 3TSS or 5TNS, but flanked by either the vector fragment VF2 or by an XhoI site plus VF2. This theme is theoretically impossible because polyadenylated mRNAs were captured for reverse transcription reaction during the cDNA library construction in terms of the protocol shown in Figure 1. Among the most frequent patterns, Case No. 5 (1.55\%, Additional file 1: Figure S1 H) indicates that designated $5^{\prime}$-end ESTs possess $3{ }^{\prime}$-end like
cDNA terminal structures. While the frequency distributions listed here are based on the UGALAB ESTs, an analysis of ESTs from NCSUFBG or TIGR_JCVIJTC leads to similar results.

\section{Restriction Enzyme Cutting Abnormality (RECA)}

Because of low frequencies in occurrence, a number of more complicated abnormal patterns of cDNA termini are not listed in Table 1. Among them, interestingly, is a set of patterns that reveal Restriction Enzyme Cutting Abnormality (RECA), as summarized in Table 2 and shown in Figure 2. It is known that, for a given cDNA library construction protocol using a specific plasmid vector (see Figure 1 for an example), the vector sequence between the two restriction enzyme (e.g., EcoRI and XhoI) recognition sites should be completely removed prior to the concatenation of a cDNA insert. However, our pattern analysis approach revealed that many variants of cDNA terminus structure patterns can indicate possible wet-lab abnormalities during the restriction enzyme digestion procedure.

\section{RECA-Type A: EcoRl site is cut off but Xhol site remains intact}

RECA-Type A is the case where the EcoRI restriction enzyme site is cut successfully by an endonuclease (restriction enzyme) while the XhoI site is kept intact. As a result, the vector fragment between the two recognition sites incorrectly remain in the vector, and the cDNA

Table 2 Summary of restriction enzyme cutting abnormality (RECA)

\begin{tabular}{|c|c|c|c|c|}
\hline Type & Feature & Pattern & $\begin{array}{l}\text { cDNA contained in } \\
\text { the sequence }\end{array}$ & Example (Additional file 1: Figure S2) \\
\hline \multirow[t]{3}{*}{$\bar{A}$} & A1: cDNA is inversely inserted & $\begin{array}{l}\text { 5': N,3TNS-1,V,3TSS-2 } \\
\text { 3': 5TNS-2,V,5TSS-1,N }\end{array}$ & $\begin{array}{l}\text { 5': a cDNA non-sense strand } \\
\text { 3': a cDNA sense strand }\end{array}$ & $\begin{array}{l}\text { FLD1_38_A06.g1_A029 (Fig. S2 A) } \\
\text { RTDR3_19_H01.b1_A022 (Fig. S2 B) }\end{array}$ \\
\hline & $\begin{array}{l}\text { A2: CDNA is inserted without } \\
\text { inversion }\end{array}$ & $\begin{array}{l}\text { 5': 5TSS,N,V,3TSS-2 } \\
\text { 3': 5TNS-2,V,N,3TNS }\end{array}$ & $\begin{array}{l}\text { 5': a cDNA sense strand } \\
\text { 3': a cDNA non-sense strand }\end{array}$ & $\begin{array}{l}\text { RTDR1_20_F07.g1_A015 (Fig. S2 C) } \\
\text { RTMNUT1_27_H12.g1_A029 (Fig. S2 D) } \\
\text { STRS1_37_H01.b1_A034 (Fig. S2 E) }\end{array}$ \\
\hline & $\begin{array}{l}\text { A3: Adapter/linker fragments } \\
\text { are inserted }\end{array}$ & $\begin{array}{l}\text { 5':5TSS,3TNS-1,V,3TSS-2 } \\
\text { 3':5TNS-2,V,5TSS-1,3TNS }\end{array}$ & No cDNA & NXRV076_A06_F (Fig. S2 F) \\
\hline \multirow[t]{2}{*}{ B } & B1: cDNA is inversely inserted & $\begin{array}{l}\text { 5': 5TSS-2,V,5TNS-1,N } \\
\text { 3': N,3TSS-1,V,3TNS-2 }\end{array}$ & $\begin{array}{l}\text { 5': a cDNA non-sense strand } \\
\text { 3': a cDNA sense strand }\end{array}$ & $\begin{array}{l}\text { NXRV_013_E07_F (Fig. S2 G) } \\
\text { RTDS1_2_A09.b1_A015 (Fig. S2 H) }\end{array}$ \\
\hline & $\begin{array}{l}\text { B2: cDNA is inserted } \\
\text { (non-inversely) }\end{array}$ & $\begin{array}{l}\text { 5': 5TSS-2,V,N,3TSS } \\
\text { 3': 5TNS,N,V,3TNS-2 }\end{array}$ & $\begin{array}{l}\text { 5': a cDNA sense strand } \\
\text { 3': a cDNA non-sense strand }\end{array}$ & $\begin{array}{l}\text { RTFEPL1_26_F12.g1_A029 (Fig. S2 I) } \\
\text { RTFEPL1_26_F12.b1_A029 (Fig. S2 J) }\end{array}$ \\
\hline C & $\begin{array}{l}\text { Neither of the two enzyme } \\
\text { sites is cut off. }\end{array}$ & $\begin{array}{l}\text { 5': 5TSS-2,V,3TSS-2 } \\
\text { 3': 5TNS-2,V,3TNS-2 }\end{array}$ & No cDNA & $\begin{array}{l}\text { NXCl_011_D03_F (Fig. S2 K) } \\
\text { NXCI_029_D07_F (Fig. S2 L) }\end{array}$ \\
\hline D & $\begin{array}{l}\text { Both the two enzyme sites } \\
\text { are cut off, but the vector } \\
\text { fragment that should be } \\
\text { removed still remains. }\end{array}$ & $\begin{array}{l}\text { 5': N,3TNS-1,V,5TNS-1,N } \\
\text { 3': N,3TSS-1,V,5TSS-1,N }\end{array}$ & $\begin{array}{l}5 \text { ': two CDNA non-sense strands } \\
3^{\prime}: \text { two } C D N A \text { sense strands }\end{array}$ & RTCNT1_24_B05.g1_A029 (Fig. S2 M) \\
\hline $\mathbf{E}$ & $\begin{array}{l}\text { Xhol cuts off at wrong } \\
\text { site from the vector. }\end{array}$ & $\begin{array}{l}\text { 5': N,V,3TSS-2 } \\
\text { 3': 5TNS-2,V,N }\end{array}$ & $\begin{array}{l}\text { 5': a cDNA sense strand } \\
\text { 3': a cDNA non-sense strand }\end{array}$ & COLD1_26_G12.b1_A029 (Fig. S2 N) \\
\hline $\mathbf{F}$ & $\begin{array}{l}\text { EcoRl cuts off at wrong site } \\
\text { from the vector. }\end{array}$ & $\begin{array}{l}5^{\prime}: \mathrm{V}, \mathrm{N} \\
3^{\prime}: \mathrm{N}, \mathrm{V}\end{array}$ & $\begin{array}{l}\text { 5': a cDNA sense strand } \\
\text { 3': a cDNA non-sense strand }\end{array}$ & RTCA1_14_E09.g1_A029 (Fig. S2 O) \\
\hline
\end{tabular}




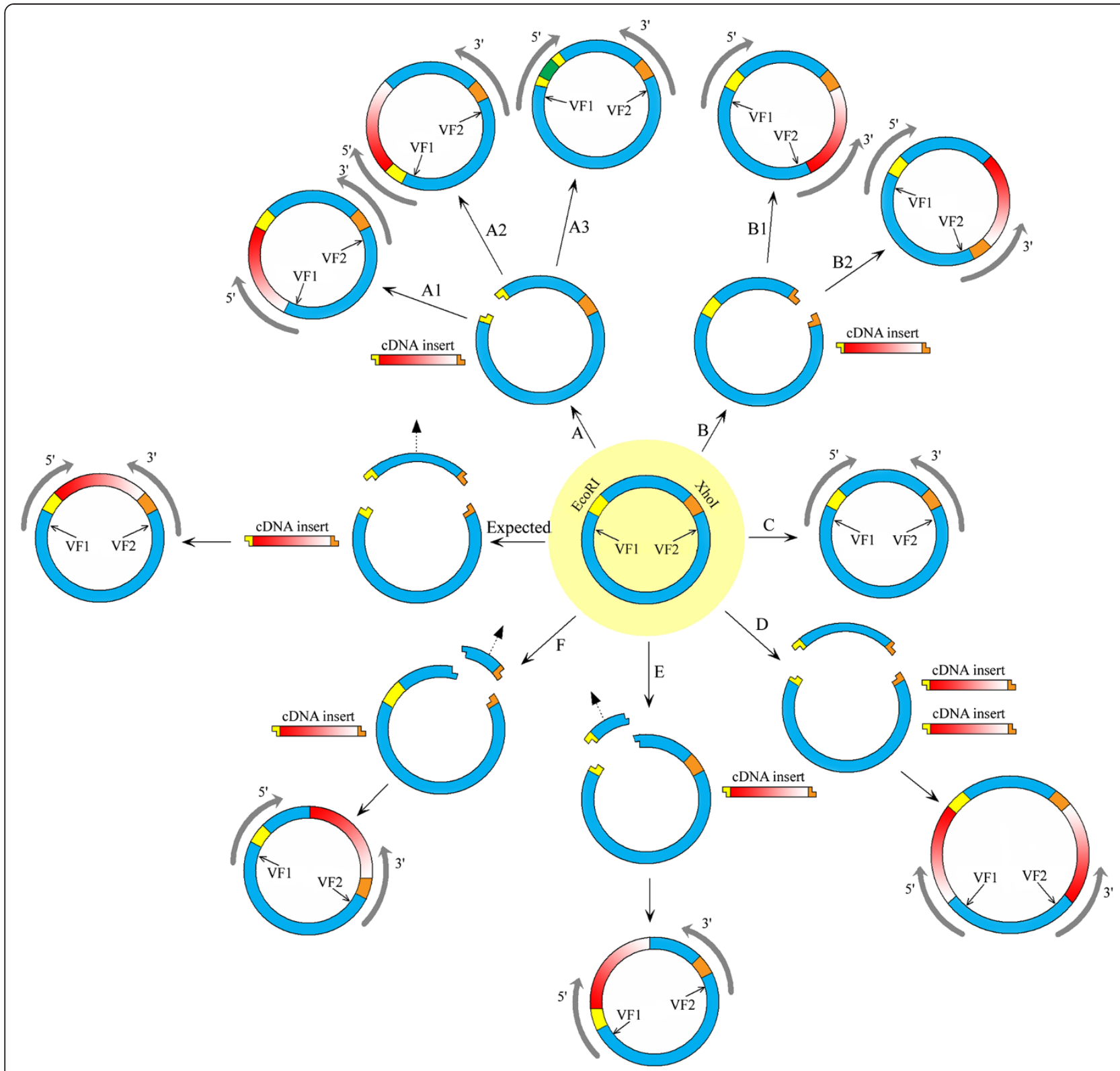

Figure 2 The expected construction of cDNA insertion and all types of Restriction Enzyme Cutting Abnormality (RECA). The label "Expected" means the expected construction of cDNA library. Sequencing direction is indicated as 3' or 5' with an arrow. VF1 (Vector fragment 1) and VF2 (Vector fragment 2) are referred to the left and right vector borders of the cloning sites. A, B, C, D, E and F are special types of RECA, defined as following: RECA-Type A: EcoRl site is cut off but Xhol site remains intact. A1: CDNA is inserted with inversion; A2: CDNA is inserted without inversion; A3: Adapter/linker fragments are inserted. RECA-Type B: Xhol site is cut off but EcoRl site remains intact. B1: CDNA is inserted with inversion; B2: CDNA is inserted without inversion. RECA-Type C: Neither of the two enzyme sites is cut off. RECA-Type D: Both the two enzyme sites are cut off, but the excised vector fragment remains. RECA-Type E: Xhol cuts off the vector at wrong site. RECA-Type F: EcoRl cuts off the vector at wrong site. The yellow color indicates EcoRI recognition site or EcoRI sticky end. The brown color stands for Xhol recognition site or Xhol sticky end. The blue represents the plasmid vector. Dark green denotes for adapter/linker fragment. cDNA insert direction is represented by red color with gradual changes: CDNA sense strand is from deep red to light red whereas cDNA non-sense strand is from light red to deep red.

inserts can be inserted into the vector by ligating to the two sticky ends of the EcoRI cut-off site. Depending on how a cDNA insert is ligated to the resultant sticky ends, we can identify A1 and A2 sub-categories, as shown in Figure 3 . In addition, we found an additional special case in which the adapter/linker fragments replace the cDNA 


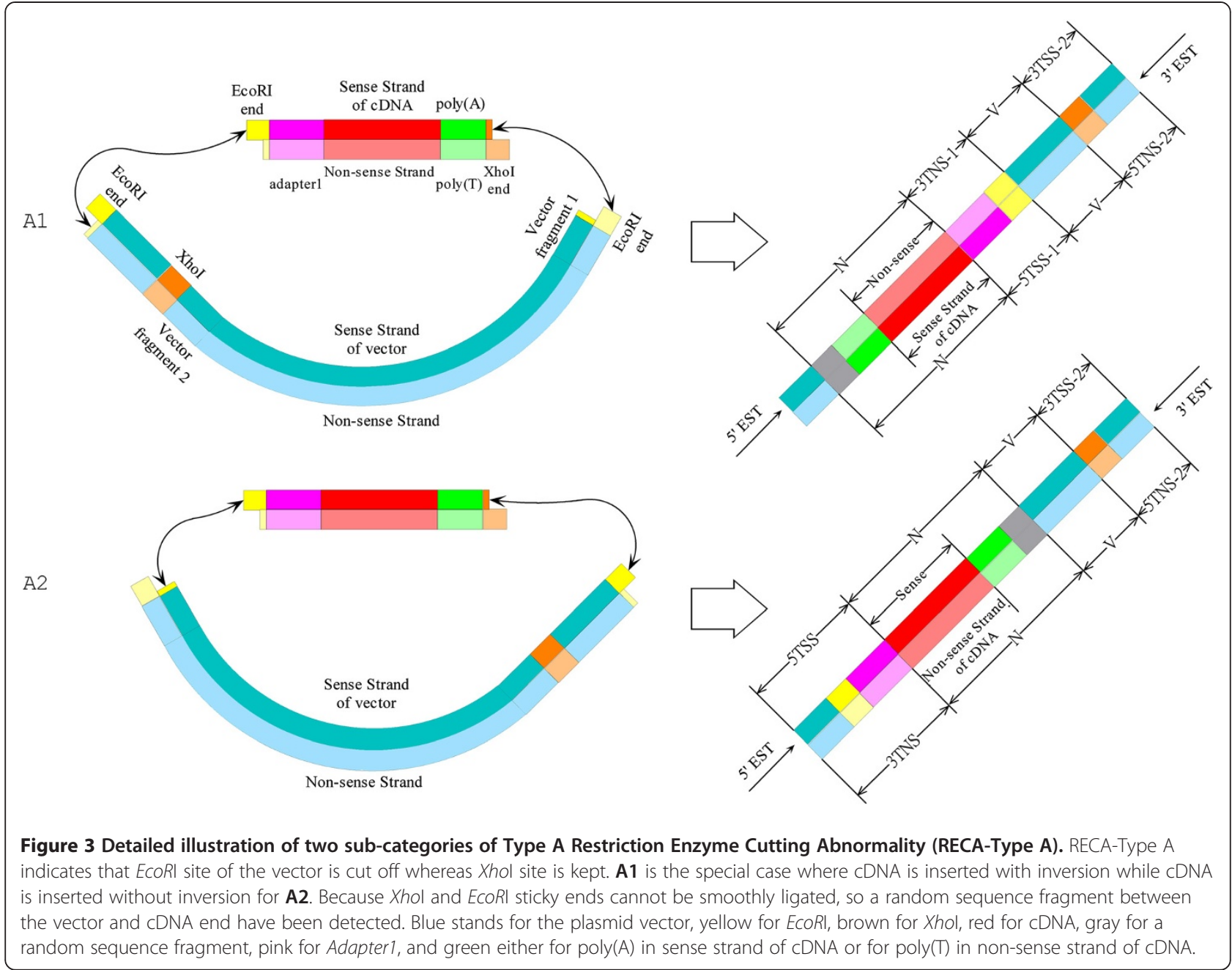

insert in the ligation. This case is categorized as A3 subcategory and shown in Figure 2.

A1: The double-stranded cDNA insert is inserted with inversion As shown in Figure 3, the double-strand cDNA insert is concatenated by the sticky ends of the vector in such a way that the sense strand (i.e., the one containing a poly(A) tail) and non-sense strand (i.e., the one containing a poly(T) tail) of the cDNA are ligated to non-sense and sense strand of the double-strand plasmid vector respectively. Consequently, the 5 '-end sequence contains cDNA sequence in the non-sense strand with a cDNA terminal pattern like $N+3 T N S$ $1+V+3 T S S-2$, whereas the 3 '-end sequence possesses cDNA sequence in the sense strand with a cDNA terminal pattern like $5 T N S-2+V+5 T S S-1+N$. Here, $V$ stands for vector fragment sequence and $N$ for non-vector sequence. As shown in Additional file 1: Figure S2 A and B, the 5'-end sequence FLD1_38_A06.g1_A029 and 3 '-end RTDR3_19_H01.b1_A022 exemplify this case. The $5^{\prime}$ end EST FLD1_38_A06.g1_A029 actually contains a 3'- end like cDNA sequence in the non-sense strand with a detected pattern of $N+3 T N S-1+V+3 T S S-2$, whereas 3'-end EST RTDR3_19_H01.b1_A022 possesses a 5' end like cDNA sequence in the sense strand with a detected pattern of $5 T N S-2+V+5 T S S-1+N$. In the GenBank submission, FLD1_38_A06.g1_A029 has not been trimmed of its 3TNS-1 component (i.e. CCTCGTGCC Adapter1 and GAATTC - EcoRI site) at the end (http://www.ncbi.nlm.nih.gov/nucest/48933478). More importantly, it has been incorrectly designated as a $5^{\prime}$-end sequence while it actually represents a 3 '-end sequence.

A2: The double-stranded cDNA insert is inserted without inversion In contrast to the aforementioned A1 sub-category, A2 represents the case where the sense and non-sense strands of the cDNA are ligated to the sense and non-sense strands of the plasmid vector at the EcoRI cut-off site - an insertion without inversion. Consequently, the resultant cDNA terminal pattern of $5^{\prime}$ end ESTs is $5 T S S+N+V+3 T S S-2$ (e.g. RTDR1_20_F07. 
g1_A015 and RTMNUT1_27_H12.g1_A029 in Additional file 1: Figure S2 C and D), whereas it is $5 T N S-2+V+N+$ $3 T N S$ for 3'-end ESTs (e.g. STRS1_37_H01.b1_A034 in Additional file 1: Figure S2 E). As shown in Figure 3, after the vector is cut off at EcoRI restriction enzyme site, there are two resultant EcoRI sticky ends available in two ends of the plasmid vector (i.e. the yellow parts in the beginning and at the end of the vector). One of these sticky ends can ligate to the counterpart EcoRI sticky end of the cDNA insert (i.e. the yellow part in the front of the cDNA insert), and the other one should be available to ligate to the XhoI sticky end of the cDNA insert (i.e. the brown part at the end of the cDNA insert). However, the EcoRI and XhoI sticky ends cannot be ligated naturally and smoothly together. Owing to this incompatibility, interestingly, we have found that some uncertain random sequences, denoted by gray color in Figure 3, have been generated during the ligation between the EcoRI and XhoI sticky ends.

A3: adapter/linker fragments are inserted Instead of a cDNA insert, adapter/linker fragments are found in some sequences to be ligated at the EcoRI cut-off site to the vector. In the example shown in Additional file 1: Figure S2 F, the 5 ' -end sequence NXRV076_A06_F displays the cDNA terminal pattern of $5 T S S+3 T N S-1+V+3 T S S-2$, and there is no cDNA insert detectable around EcoRI cut-off site. Unfortunately, in the GenBank submission (http:// www.ncbi.nlm.nih.gov/nucest/21689178), the region between 157 and 432 was taken as the final clean sequence, which was identified as a vector fragment sequence using our method.

\section{RECA-Type B: the Xhol site is cut off while the EcoRl site remains intact}

In Figure 2, similar to the RECA-Type A, there are two sub-categories whose definitions are based on whether or not the cDNA insert is inversely ligated at the XhoI cut-off site: B1 is for the insertion (ligation) with inversion (see NXRV_013_E07_F and RTDS1_2_A09.b1_A015 in Additional file 1: Figure S2 G and $\mathrm{H}$ for examples) and B2 for the insertion (ligation) without inversion (see RTFEPL1_26_F12.g1_A029, and RTFEPL1_26_F12.b1_A029 in Additional file 1: Figure S2 I and J for examples).

\section{RECA-Type C: Neither of the enzyme sites is cut off}

As shown in Figure 2, sometimes neither of the two restriction enzymes is successfully cut from the vector and consequently no cDNA fragment is inserted. This case is exemplified by NXCI_011_D03_F and NXCI_029_D07_F in Additional file 1: Figure S2 K and L, both of which have a terminal pattern of $5 T S S-2+V+3 T S S-2$.
RECA-Type D: Both EcoRI and Xhol sites are cut off, but the vector fragment that theoretically should be removed still remains

This type is a combination of RECA-Type A1 and B1. The abnormality appears to be caused by the fact that, after the cutoff at both EcoRI and XhoI sites, two cDNA inserts are inserted or ligated at both cutoff sites separately. Consequently, no vector fragment is actually cut off and removed (see Figure 2 D). Depending on the orientation of the two cDNA inserts, there are a number of complex sub-categories. Currently, one relevant terminal pattern that has been detected is $N+3 T N S$ $1+V+5 T N S-1+N$, exemplified by $5^{\prime}$-end sequence RTCNT1_24_B05.g1_A029 in Additional file 1: Figure S2 $\mathrm{M}$.

\section{RECA-Type E: The restriction enzyme Xhol did not cut at its recognition site}

The vector sequence fragment between the restriction enzyme recognition sites of EcoRI and XhoI should theoretically be removed from the vector, but our analysis shows that part of this vector fragment flanking XhoI recognition site still remains in some ESTs. One reasonable explanation of this phenomenon is that the restriction enzyme XhoI failed to cut off the vector at its recognition site (see Figure 2, Type E). As shown in Additional file 1: Figure S2 N, COLD1_26_G12.b1_A029 appears to represent this case, in which a 3 '-EST displays the cDNA terminal pattern as $5 T N S-2+V+N$.

\section{RECA-Type F: The restriction enzyme EcoRI did not cut at its recognition site}

Similar to RECA-Type E, when the restriction enzyme fails to cut the vector at the EcoRI site, part of the vector fragment flanking EcoRI site can be retained in some ESTs (see Figure 2, Type F). As shown in Additional file 1: Figure S2 O, RTCA1_14_E09.g1_A029 supports this case, showing that a $5^{\prime}$-EST can have a cDNA terminal pattern like $V+N+3 T S S$. 3TSS is not necessarily detectable due to either low quality sequence region or longer cDNA insert.

Overall, we detected 1,087 EST sequences with RECA cases. Summing up all sequence numbers of each RECA types in Table 3, we can see that RECA-Type A (52.8\%), RECA-Type B (21.8\%) and RECA-Type C (23.0\%) are the most common types. RECA-Type D, E and F account for about $1.7 \%$ of all cases, whereas all other unclassified, complicated cases make up about $0.6 \%$. Of all the three labs, UGALGB has 765 RECA sequences and NCSUFBG has 322, whereas no RECA case is detected in the ESTs from TIGR_JCVIJTC. Interestingly, different labs have different RECA types. For example, RECAType A2, D, E and F are found in UGALAB ESTs but not in NCSUFBG ESTs whereas RECA-Type A3 and C 
Table 3 Numbers of each type of RECA sequences

\begin{tabular}{|c|c|c|c|c|c|c|c|c|c|c|c|}
\hline & \multicolumn{10}{|c|}{ RECA Type } & \multirow{2}{*}{$\begin{array}{l}\text { Total } \\
\text { Sequence }\end{array}$} \\
\hline & $\overline{\mathrm{A} 1}$ & $A 2$ & $A 3$ & B1 & B2 & $\mathrm{C}$ & D & $E$ & $F$ & Other $^{1}$ & \\
\hline UGALAB & 432 & 101 & 0 & 107 & 100 & 0 & 2 & 9 & 9 & 5 & 765 \\
\hline NCSUFBG & 3 & 0 & 37 & 18 & 12 & 250 & 0 & 0 & 0 & 2 & 322 \\
\hline Total & 436 & 101 & 37 & 125 & 112 & 250 & 1 & 9 & 9 & 7 & 1087 \\
\hline
\end{tabular}

${ }^{1}$ Other types include two cases: (1) sequences with complicated patterns whose type is hard to be determined; (2) sequences with too bad quality to determine the sequence type

are identified in NCSUFBG ESTs but not in UGALAB ESTs.

Because the bona fide cDNA fragments in the raw sequences with RECA cases are difficult to determine unambiguously, these ESTs should be filtered out and subjected to further scrutiny before their deposition into the public databases like GenBank. Unfortunately, most of them have been submitted to GenBank by conventional EST processing pipelines that do not examine cDNA termini and their variations (Additional file 1: Figure S2 A-J, M-O).

\section{Double-Termini Adapter (DBT)}

Previously, we reported abnormal ESTs with double-termini adapters (DBT) - a palindrome linker, made from two mutually exclusive terminus components (e.g., the adapter 5' -CCTCGTGCC- 3' from 3TNS, the EcoRI site $5^{\prime}$-GAATTC- 3' from either 3TNS or 5TSS, and the adapter 5' -GGCACGAGG- 3' from 5TSS, where 5TSS and 3TNS should be, in theory, mutually exclusive as per Figure 1), that could bring separate $3{ }^{\prime}$ and 5 ' directional sequence fragments together into a single, chimeric EST sequence [10,11]. Detailed pattern analysis of these DBT ESTs reveals two distinct sub-categories, which we call Type 1 Concatenation and Type 2 Concatenation, and note that Type 2 Concatenation is a novel finding in this study. As shown in Figure 4, Type 1 Concatenation possesses a sequence pattern of CCTCGTGCC + G + AATTC + GGCACGAGG, whereas Type 2 Concatenation has AATTC + GGCACGAGG + CCTCGTGCC + G. Type 2 concatenation indicates that Adapter 1 in the sense strand can be connected directly to Adapter1 in the nonsense strand. Among 309,976 raw Pinus taeda ESTs, we found that $3.5 \%$ of the UGALAB ESTs $(6,045$ out of 172,229 ) and $0.9 \%$ of the NCSUFBG ESTs (681out of 75,001) have Type 1 Concatenation, while 2,515 from UGALAB and 741 from NCSUFBG possess Type 2 Concatenation. It is interesting to note that many ESTs have continuous, repetitive and mixed Type 1 and Type 2 Concatenations, as shown in Additional file 1: Figure S3 A and B. How many repetitions of such combined concatenations exist in a sequence? This might be a random effect involving complex biochemical reactions, because we have uncovered single-, double- and triple-DBT repetitions. Because of such complex concatenations, we might not be able to conclude that all DBTs will bring $3^{\prime}$ and $5{ }^{\prime}$ directional sequences to form a single chimeric EST. For example, as shown in Additional file 1: Figure S3 B, FLD1_32_F06.b1_A029 has a complex concatenated adapter immediately before the 3TNS terminus:

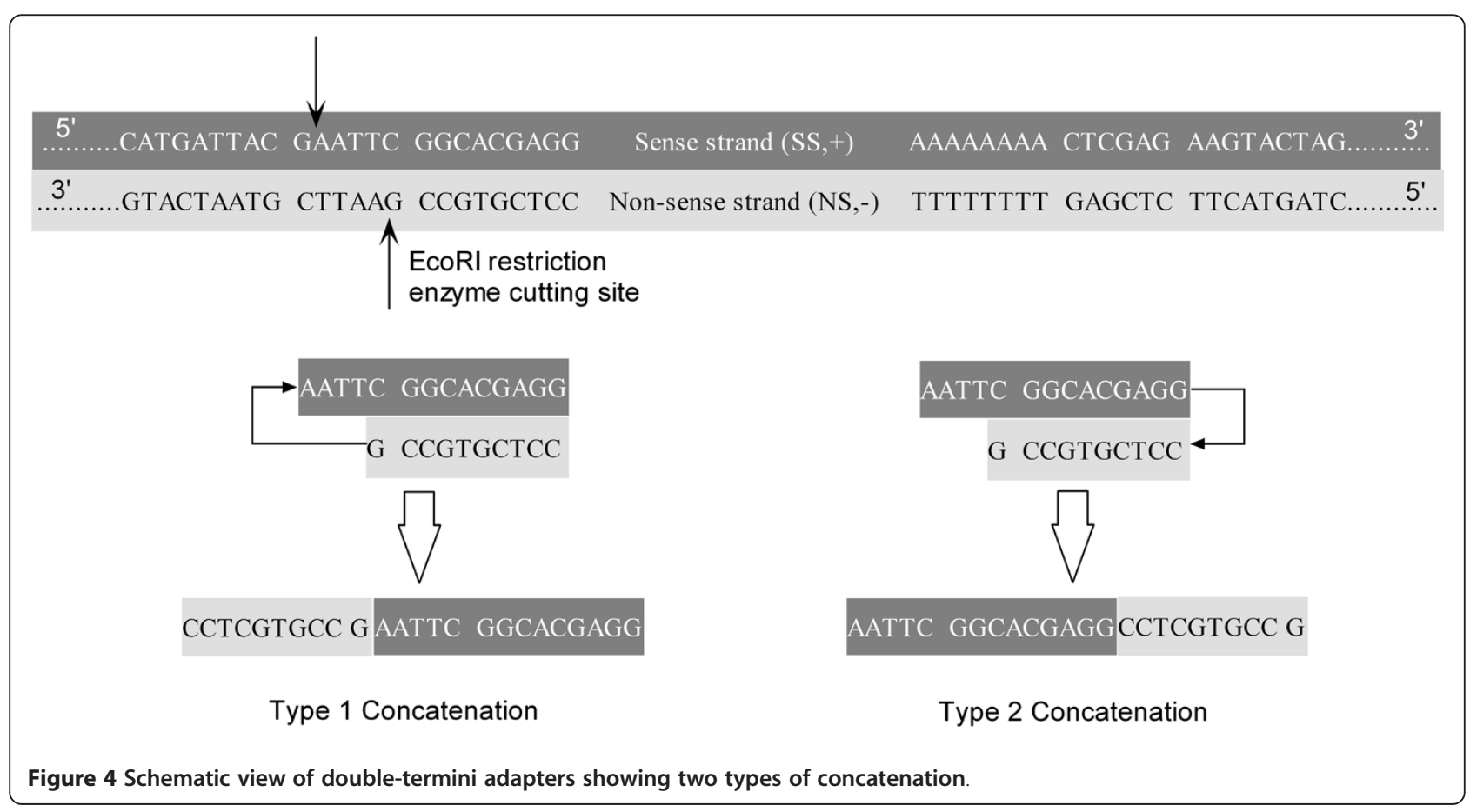


CCTCGTGCC (Adapter1 in non-sense direction) is connected to GAATTC (EcoRI site); GAATTC is concatenated to GGCACGAGG (Adapter1 in sense direction); GGCACGAGG is concatenated with CCTCGTCC again, which is part of the normal 3TNS terminus. In this case, all of the sequences should belong to 3 '-directional sequences, not chimeric sequences at all. In fact, about $0.24 \%$ of 3 '-end sequences of UGALAB have the pattern $5 T N S+N+D B T+3 T N S$ with this feature. When processing this kind of sequence, it is appropriate to take only the region between 5TNS and 3TNS, excluding DBT, as the cDNA inserts. However, current processing pipelines have overlooked this abnormal terminus and submitted sequences including DBT to GenBank (Additional file 2: Table S1).

\section{Our software tool - AFST}

Current EST processing pipelines that do not examine cDNA terminal structures apparently do not have the ability to detect and filter aforementioned abnormal sequences properly before the GenBank dbEST submission. Using pattern analysis, we have created a unique EST processing protocol to determine the bona fide cDNA inserts within raw EST sequence reads generated by Sanger sequencing. Based on this protocol, we developed a software tool called AFST (Abnormality Filtering and Sequence $\underline{T}$ rimming for raw ESTs) that can identify cDNA terminal structures, visualize sequence abnormalities, and trim ESTs properly. As an open-source tool, the executable and source codes of AFST are available online (http://code.google.com/p/afst).

Implemented in Java with a MySQL or SQLite backend database, AFST allows users to load their raw ESTs in FASTA format, with or without a relevant quality file, and specify the vector sequence, adapter sequence(s), and the restriction enzyme recognition sites adopted in their cDNA library construction protocols. After execution, as shown in Figure 5A, AFST is able to provide a tabular result showing final clean, trimmed sequences and information about sequence abnormality such as DBT and RECA. Moreover, AFST can provide more detailed information about the cDNA termini (Figure 5B) and abnormalities detected (Figure $5 \mathrm{C}$ ) for each individual sequence, and allow users to export results selectively for further data analysis. Besides the GUI version, we also create a command line version of AFST that can be easily integrated into existing bioinformatics pipelines for automatic data processing.

In order to compare AFST with other EST cleanup tools or pipelines, we compared Genbank ESTs for two species with our processing results because these Genbank ESTs have been trimmed by other tools or pipelines. Of the 309,976 raw Pinus taeda ESTs, we carefully examined 230,783 GenBank ESTs that had been submitted by at least three different EST processing pipelines $[1-3,10]$. We found that among them $5.2 \%$ (i.e., 11,986 ESTs) are "unclean" and 2.2\% (i.e., 5,078 ESTs, including 3,180 that have DBTs) are abnormal, both of which could be cleaned or filtered by AFST. Moreover, two other popular tools for raw Sanger EST cleanup and trimming - Lucy [13,14] and SeqClean [15] were adopted to process the 309,976 raw ESTs. Interestingly, among the final clean ESTs trimmed by Lucy, 55,993 $(18.1 \%$ of 309,976$)$ are still unclean, $3,789(1.22 \%$ of 309,976) have DBTs, and of all the 1,087 RECA sequences, 771 ESTs $(70.9 \%$ of 1,087$)$ are unidentified. Meanwhile, among the final clean ESTs trimmed by SeqClean, $33,494(10.8 \%$ of 309,976$)$ are still unclean, 6,416 $(2.1 \%$ of 309,976$)$ have DBTs, and of all the 1,087 RECA sequences, 934 ESTs $(85.9 \%$ of 1,087$)$ are undetected. In order to demonstrate that our protocol performs well for cDNA libraries other than those in Pinus taeda, we reprocessed 38,709 peanut (Arachis hypogaea) ESTs from GenBank dbEST that utilizes pBluescript II SK as the vector and EcoRI and XhoI as the two restriction sites. Consequently, we found 25.3\% (i.e., 9,785 ESTs) are "unclean" and 3.9\% (i.e., 1,510 ESTs, including 259 that have DBTs) are abnormal using AFST.

\section{Conclusions}

The ever-growing collection of EST sequences in GenBank is an important bioinformatics resource, providing crucial data for downstream applications related to gene identification, functional annotation, SNP and other polymorphism identification, and so on. Providing clean data is crucial if these applications are to be used to correct analysis. Although there have been significant efforts to filter error-prone ESTs, many GenBank ESTs are still problematic. As demonstrated in both this and previous studies $[10,11]$, current bioinformatics protocols and approaches do not explore the essence of potential EST data abnormalities from the perspective of cDNA library construction. Consequently, they have deposited a significant amount of unclean and abnormal ESTs into the public repositories and created potential problems for data-dependent downstream applications. Without inspecting cDNA terminal structures, existing EST processing programs fail to achieve sufficient data quality control and are unlikely to identify and remove common error-prone ESTs before GenBank deposition. In this study, we have adopted a novel pattern analysis approach that proves to be effective in identifying and distinguishing EST sequence abnormalities based on cDNA termini structures. This approach improves identification accuracy of the bona fide start and stop position of a cDNA insert within a raw EST sequence, thus significantly improving EST data quality. It also helps illustrate wet-lab abnormalities that can reveal potential 


\section{a}

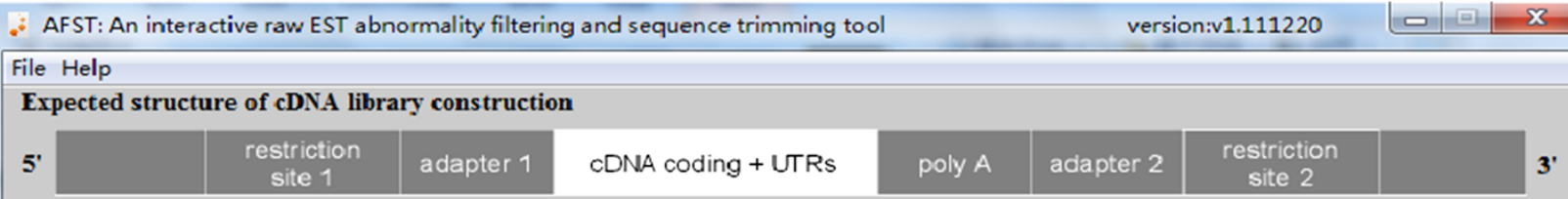

Specify the vector, restriction sites and adapter(s) used in the cDNA library construction.

vector file emp/sampleData/vect or/pSL1180. complete. fasta

sequence file temp/sampleData/seqData/1000. fasta

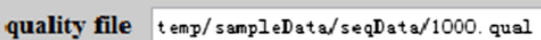

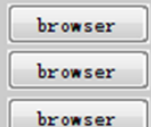

by sequence name -

$\square$ no quality file

ErrorBase

\begin{tabular}{rl|l|} 
restriction site $\mathbf{l}\left(\mathbf{5}^{\prime}-\mathbf{3}^{\prime}\right)$ & GAAIIC & 1 \\
\hline restriction site $\mathbf{2}\left(\mathbf{5}^{\prime}-\mathbf{3}^{\prime}\right)$ & CICGAG & 1 \\
\hline adapter $\mathbf{l}\left(\mathbf{5}^{\prime}-\mathbf{3}^{\prime}\right)$ & GGCACGAGG & 1 \\
\hline
\end{tabular}

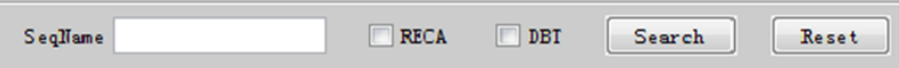

\begin{tabular}{|c|c|c|c|c|c|c|c|c|}
\hline 1 & GO & & & & $\mathbb{A} \triangle D D$ & & displs & Page 1 of 100 \\
\hline$\#$ & Seqliane & ESI Dir & DBI & RECA & Abnormal Iag & FinalSeq Start & Finalseq End & Finalseq Len \\
\hline 1 & COLD1_14_C01.b1_AO29 & 3 & $\square$ & $\square$ & $\square$ & 4 & 619 & 616 \\
\hline 2 & RTDR3_19_H01.b1_A022 & 3 & $\square$ & $\nabla$ & $\nabla$ & -1 & -1 & 0 \\
\hline 3 & COLD1_14_E10.G1_AO29 & 5 & $\square$ & $\square$ & $\square$ & 43 & 483 & 441 \\
\hline 4 & RICII1_24_B05. G1_A029 & 5 & $\square$ & $\nabla$ & $\nabla$ & -1 & -1 & 0 \\
\hline 5 & COLD1_15_E08. b1_AO29 & 3 & $\square$ & $\square$ & $\square$ & 27 & 734 & 708 \\
\hline 6 & COLD1_15_F01.b1_A029 & 3 & $\nabla$ & $\square$ & $\nabla$ & -1 & -1 & 0 \\
\hline 7 & COLD1_13_A03. b1_AO29 & 3 & V & $\square$ & v & -1 & -1 & 0 \\
\hline 8 & COLD1_18_H08.b1_AO29 & 3 & $\nabla$ & $\square$ & $\nabla$ & -1 & -1 & 0 \\
\hline 9 & COLD1_19_C09.b1_A029 & 3 & $\square$ & $\square$ & $\square$ & 55 & 294 & 240 \\
\hline 10 & RIDS3_1_G07.b2_A022 & 3 & $\square$ & $\nabla$ & $\nabla$ & -1 & -1 & 0 \\
\hline
\end{tabular}

b

\section{>NDL1_11_A06.b1_A029 3'EST}

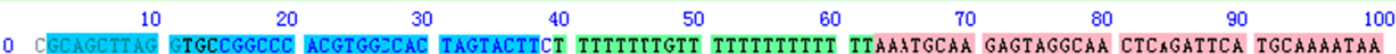

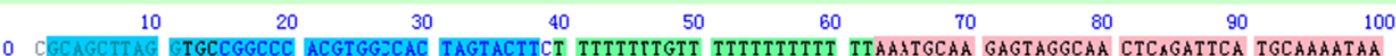

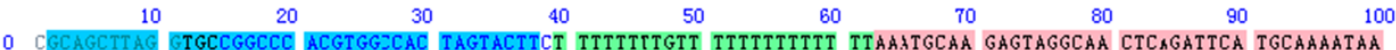

$\checkmark$ Space Seperator RECA Type:

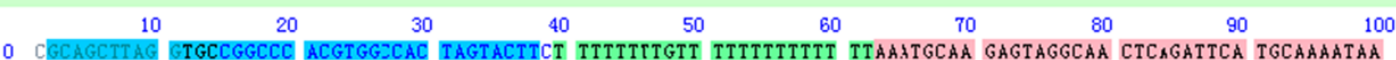

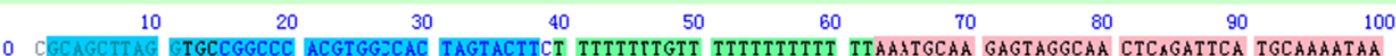

Execute

SampleD ata

Execute

1 AAATATTTAT TACATAGAGA TTGAGTZATT TGCTATTAAA TGACATGTAT CCCACCCTAT TTGCTATCCT GTGAAAAATC TAACAAAATG GAAAGAAAAT

2 ACGCCATCCA GCACTAGGAT GAGCTTICTC TCAAATTAAA ATTTCAGAGC AAATCTATGC TAAGATTTGT TTAGCATTTG CAACCACATT GTCAGCAGTG

3 AGGCCAAACT CCTTGTACAA GATTCCAATG GGAGCACTCG CACCAAACCT GTCTATCCCA ACAGCCTTTC CCTTGGATCC TAAGTACCTC TCCCAACCAA

4 AAGTTGAACC AGCTTCCACA CTGACCZTTG CAGCAACAGC TGCCGBAAGC ACACTTTCTT TATATGCTGG AGTTTGTTCC TCGAATAATT CCCAACAGAC

5 AAGGGAACA ACTCTAACAG CTTTCCZTTC ATTCCTCAAA GTAGATGCAG CTTTCTCAGC AATCTCAAGC TCAGAACCAC TACCCATAAG ÄATACATCA

6 GGCTTGTTGC CAGAGGAATT GTCACTJATA ATGTAGCCTC CCTTCTCGAC ACCCTCTATT GAAAACCGGC AAGATGTGGG AGT:TTTGAC GGGAGAGAGC

7 AAGTATTGAT GGCCTCTTTC TATTGAJAAC AGCAACCTTG TAAGCCCCAG CTGTCTCCTT TCCATCAGCT GGACGGACA TCAAACATT GGGCATTGCC

8 CCTAAAGTT GCAAGTGCTC TATTGGäTG ATGAGTGGGC CCATHICTCT CGAGACCAAT HGASCATGC GTCATTACAT AUGATACACA GUCTTTCAGC

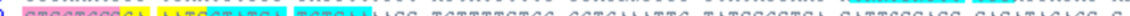

\section{C}

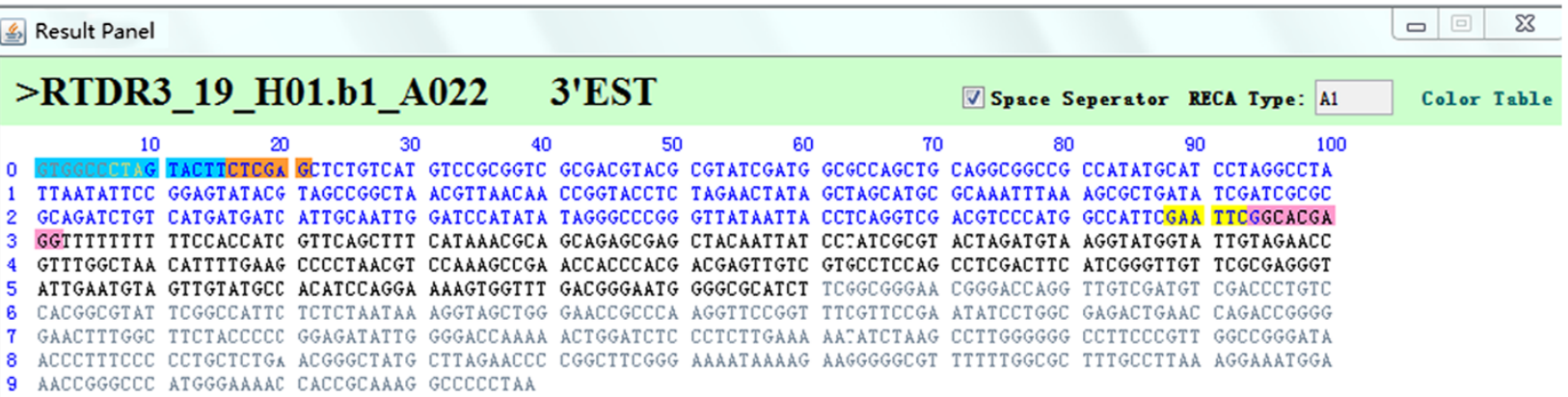

Figure $\mathbf{5}$ (See legend on next page.) 
(See figure on previous page.)

Figure 5 Snapshots of AFST user interfaces. a: The main interface allows users to upload their sequences, specify relevant information about vector and adapter/linker sequences, initiate data processing, and obtain tabular results showing abnormality. b: Details of a normal sequence. The high-quality region between 5TNS-4 (from 2 to 62, marked with blue and green) and 3TNS (from 900 to 926, marked with pink, yellow and blue) is the final clean sequence (i.e., the region with a light red background). The color legends and their meanings can be found by clicking 'color table'. c: Details of an abnormal sequence. This sequence has RECA abnormality (RECA-Type A1), where the double-stranded cDNA insert is inverted in its orientation and inserted into the double-strand plasmid vector after enzyme digestion. The vector sequence region between 5TNS2 (highlighted with blue and brown) and 5TSS-1 (highlighted with yellow and pink) is the part that should have been cut off theoretically after enzyme digestion.

error sources, such as a failure of one or both of the restriction enzymes to cut the plasmid vector, a failure of the restriction enzymes to cut the vector at the correct positions, the insertion of two cDNA inserts into a single vector, the insertion of multiple and/or concatenated adapter links, the presence of 3 '-end terminal structure in designated $5^{\prime}$-end sequences and vice versa, and so on. In particular, the double-termini adapter (DBT) reported previously as one EST abnormality $[10,11]$ proves to have a novel variation (i.e., the Type 2 Connection) identified by the pattern analysis approach adopted in this study. Clearly, our pattern analysis approach and the relevant software tool AFST will help biologists diagnose the potential problems in wet-lab procedures and facilitate creation of more accurate data.

\section{Methods}

We downloaded all 309,976 raw Sanger ESTs for Pinus taeda from NCBI Trace Archive (http://trace.ncbi.nlm. nih.gov/Traces/trace.cgi), which were generated by three different sequencing centers or labs including previously well-known TIGR institute. For these raw ESTs, we were able to collect the complete information about cDNA library construction protocol (i.e., plasmid vector, adapter or linker sequences, restriction enzyme sites, sequence name convention and associated sequencing directions), which is required by AFST to conduct accurate pattern analysis. In particular, 230,783 out of 309,976 ESTs were submitted by each center or lab into GenBank dbEST as final clean ESTs after raw EST cleanup and trimming. Therefore, this dataset represents a valuable benchmark for us to evaluate AFST performance. Also due to the availability of the complete cDNA library construction information, we were able to use two other popular Sanger EST cleanup tools - Lucy [13,14] and SeqClean [15] to process 309,976 raw ESTs for performance comparison with AFST. In order to demonstrate that our protocol performs well for cDNA libraries other than those in Pinus taeda, we downloaded all Arachis hypogaea (peanut) ESTs $(86,939)$ from dbEST, which were deposited by many labs and investigators. Among them, the biggest data set was 38,709 , whose complete cDNA library construction protocol information (i.e., pBluescript II SK as the plasmid vector and EcoRI and $\mathrm{XhoI}$ as the restriction enzyme sites, etc.) was available by extracting and parsing dbEST records. Because cDNA library construction information is not mandatory for dbEST submission, it is often difficult to get complete cDNA library constructional information among dbEST records. Therefore, we used AFST to process 38,709 peanut ESTs and detect cDNA terminus patterns.

In our pattern analysis protocol, there are three important concepts worthy of further explanation: Pattern, Confidence score and Reasonable pair. They are also implemented in our software tool AFST to identify abnormal sequences such as those with RECA and DBT and determine final clean ESTs.

\section{Pattern}

"Pattern" refers to a cDNA terminus structure detected in a raw EST sequence. It is determined by the type, number, order and context of all cDNA termini in terms of the specification (expectation) given by a specific cDNA library construction protocol. To identify the pattern, we first find all putative cDNA termini existing in the sequence, then consider good/low quality regions and vector fragment positions, and finally determine the pattern with respect to the following aspects:

(1)Type of cDNA termini. There are four major types of termini [5TSS], [3TSS], [5TNS] and [3TNS] as shown in Figure 1. Each of these termini can be further categorized into some sub-categories. For example, [3TSS] includes 3TSS, 3TSS-1, 3TSS-2, 3TSS-3, 3TSS-4 and 3TSS-5 (also see Figure 1).

(2) Number of cDNA termini. For instance, a sequence with just one [5TSS] and another sequence with two [5TSS] have different patterns.

(3) Sequential order of cDNA termini. For example, a sequence with first a [5TSS] and then a [3TSS] has a different pattern from another sequence that has first a [3TSS] and then a [5TSS].

(4) Context of cDNA termini. Flanking region context refers to the sequence region immediately before/ after a terminus. There are mainly two cases: vector fragment (represented by $V$ ) and non-vector fragment $(N)$. Furthermore, vector or non-vector fragments can be further classified into vector 
fragment in high quality region $(H V)$, vector fragment in low quality region $(L V)$, non-vector fragment in high quality region $(H N)$ and nonvector fragment in low quality region $(L N)$. Context is one of the basis on which terminus' confidence is estimated by computing a confidence score (see below).

\section{Confidence score}

Because of sequencing errors [16-18], some in-silico identified cDNA termini might be false positives. When a terminus defined in Figure 1 is detected, we will quantify our confidence in its detection with a confidence score, which is calculated by considering the extent of the completeness of all required sequence elements, adjacent sequence contents (contexts) and the percentage of bases that match the whole terminus.

(1)Determine the completeness score for a given terminus ( $A$ score). The completeness score is directly reflective of the number of sequence elements in the terminus. For example, the completeness score of 5TNS, which has three sequence elements (i.e., a poly $(\mathrm{T})$ tail, XhoI site, and $V F 2$ ), is higher than 5TNS-1 and 5TNS-2, each of which have only two sequence elements. Comparing with 5TNS, 5TNS-1 and 5TNS-2, the completeness score of 5TNS-3 is the lowest one because it only has one sequence element. The terminus with the higher completeness score is more likely to be authentic, instead of being an artifact of a sequencing error.

(2)Score a terminus according to its flanking region context ( $B$ score). Sequence contents that match the expected structures in terms of a cDNA library construction protocol deserve a higher score. For example, we expect to detect a vector fragment sequence immediately upstream of 5TSS. Correspondingly, the vector fragment, the lowquality non-vector fragment, and the high-quality non-vector fragment detected immediately upstream of 5TSS will result in the highest (100), intermediate (50) and lowest $B$ score (0) respectively, which will be assigned to the identified 5TSS.

(3) Score the percentage of matched bases ( $C$ score). The percentage is calculated in terms of the detected bases that are the same as the expected bases divided by length of the terminus. For example, the 3' EST NDL1_11_A06.b1_A029 in Additional file 1: Figure S3 $C$ has the cDNA terminal pattern of $5 T N S-4+N+3 T N S-$ $5+N+3 T N S+V$. Obviously the cDNA terminus detected in the front is 5TNS-4, whereas the terminus detected at the end can be either a 3TNS or 3TNS-5. Because the percentage of matched bases is much lower for 3TNS-5 than for 3TNS, as well as due to the adjacent sequence contents (contexts), 3TNS is assigned with a higher $C$ score than 3TNS-5. Therefore, we identified 3TNS as the real terminus at the end of this EST while 3TNS-5 was a false one. The formula that determines the confidence score for a given terminus is:

$$
\begin{aligned}
& \text { Confidence Score }=\text { weight } A * A \text { score } \\
& \text { + weight } B * B \text { score }+ \text { weight } C * \text { C Score. }
\end{aligned}
$$

\section{Reasonable pair of detected termini}

Based on the expected cDNA terminus structure shown in Figure 1, if the cDNA insert is short enough, we should be able to detect both the $5^{\prime}$ end terminus and the $3^{\prime}$ end terminus in an EST sequence. This also means that we are able to detect a reasonable pair of cDNA termini for some ESTs, using the following definitions: (1) for a $5^{\prime}$-end EST, [5TSS] and [3TSS] is the reasonable termini pair where [5TSS] should be upstream of [3TSS]; (2) for a 3 '-end EST, [5TNS] and [3TNS] is the reasonable termini pair where [5TNS] should be upstream of [3TNS]; (3) the distance between the two paired termini shouldn't be too short to contain a cDNA insert (i.e. $>=200$ bases).

In our software tool AFST, essentially, we first determine all cDNA terminal patterns according to the type, number, order and context of all expected termini, and identify and filter out RECA and DBT abnormalities. We then search for reasonable terminus pairs, calculate confidence scores for all detected termini, and select the reasonable terminus pair that yields the highest cumulative confidence scores to delineate the start and end positions of the bono fide cDNA insert. Finally, the final clean sequence is obtained by trimming off both lowquality regions and vector fragments from the sequence fragment between the two termini of the best reasonable pair.

\section{Additional files}

Additional file 1: Figure $\mathbf{S 1}$ Sequences with abnormal CDNA terminus structures. All the sequences mentioned in Results and discussion section (Part 1) are listed. Figure S2. All types of Restriction Enzyme Cutting Abnormity (RECA). All sequences that possess RECA and are described in Results and discussion Section (Part 2) are listed. Figure S3. Other examples of abnormal EST sequences. All other abnormal sequences discussed in this paper are listed.

Additional file 2: Table S1 Some raw EST sequences with the "5TNS + N + DBT + 3TNS" pattern had been submitted to GenBank. Most of them have their DBTs untrimmed in Genbank.

\section{Abbreviations}

5TSS: $5^{\prime}$ terminus of the CDNA in the sense strand; 3 TSS: $3^{\prime}$ terminus of the cDNA in the sense strand; 5TNS: $5^{\prime}$ terminus of the CDNA in the non-sense 
strand; 3TNS: $3^{\prime}$ terminus of the CDNA in the non-sense strand; $\mathrm{V}$ : Vector sequence, either HV or LV; N: Non-vector sequence, either $L N$ or $H N$; $H V$ : Vector fragment in high quality region; LV: Vector fragment in low quality region; HN: Non-vector fragment in high quality region; LN: Non-vector fragment in low quality region.

\section{Competing interests}

The authors declare that they have no competing interests.

\section{Authors' contributions}

GJ and CL managed and coordinated the project. SZ carried out main implementation and data analysis. XL, PL and JM contributed to some implementation. JK contributed critically to manuscript editing. All authors participated in manuscript writing and editing. All authors read and approved the final manuscript.

\section{Acknowledgements}

This project was funded by the National Natural Science Foundation of China (No. 61174161), the Specialized Research Fund for the Doctoral Program of Higher Education of China (No. 20090121110022), the Fundamental Research Funds for the Central Universities of Xiamen University (Nos. 2011121047, 201112 G018 and CXB2011035), the Key Research Project of Fujian Province of China (No. 2009H0044) and Xiamen University National 2113 rd Period Project of China (No. 0630-E72000).

\section{Author details}

'Department of Automation, Xiamen University, Xiamen, Fujian 361005 , China. ${ }^{2}$ Department of Botany, Oxford, OH 45056, USA. ${ }^{3}$ Department of Computer Science and Systems Analysis, Oxford, OH 45056, USA. ${ }^{4}$ Department of Microbiology, Oxford, OH 45056, USA. ${ }^{5}$ Department of Statistics, Miami University, Oxford, OH 45056, USA.

Received: 18 December 2011 Accepted: 15 March 2012

Published: 03 May 2012

\section{References}

1. Cairney J, Zheng L, Cowels A, Hsiao J, Zismann V, Liu J, Ouyang S, ThibaudNissen F, Hamilton J, Childs K, Pullman GS, Zhang Y, Oh T, Buell CR: Expressed Sequence Tags from loblolly pine embryos reveal similarities with angiosperm embryogenesis. Plant Mol Biol 2006, 62:485-501.

2. Lorenz WW, Sun F, Liang C, Kolychev D, Wang H, Zhao X, Cordonnier-Pratt $M M$, Pratt $L H$, Dean JF: Water stress-responsive genes in loblolly pine (Pinus taeda) roots identified by analyses of expressed sequence tag libraries. Tree Physiol 2006, 26:1-16.

3. Pavy N, Laroche J, Bousquet J, Mackay J: Large-scale statistical analysis of secondary xylem ESTs in pine. Plant Mol Biol 2005, 57:203-224.

4. Adams MD, Kelley JM, Gocayne JD, Dubnick M, Polymeropoulos MH, Xiao H, Merril CR, Wu A, Olde B, Moreno RF, et al: Complementary DNA sequencing: expressed sequence tags and human genome project. Science 1991, 252:1651-1656.

5. Adams MD, Dubnick M, Kerlavage AR, Moreno R, Kelley JM, Utterback TR, Nagle JW, Fields C, Venter JC: Sequence identification of 2,375 human brain genes. Nature 1992, 355:632-634.

6. Liang F, Holt I, Pertea G, Karamycheva S, Salzberg SL, Quackenbush J: Gene index analysis of the human genome estimates approximately 120,000 genes. Nature Genet 2000, 25:239-240.

7. Clark MS, Edwards YJ, Peterson D, Clifton SW, Thompson AJ, Sasaki M, Suzuki Y, Kikuchi K, Watabe S, Kawakami K, Sugano S, Elgar G, Johnson SL: Fugu ESTs: New resources for transcription analysis and genome annotation. Genome Res 2003, 13:2747-2753.

8. Brent MR: Steady progress and recent breakthroughs in the accuracy of automated genome annotation. Nature Rev Genet 2008, 9:62-73.

9. GenBank dbEST::; [http://www.ncbi.nlm.nih.gov/projects/dbEST/].

10. Liang C, Wang G, Liu L, Ji GL, Fang L, Liu YS, Carter K, Webb JS, Dean JFD: ConiferEST: an integrated bioinformatics system for data reprocessing and mining of conifer expressed sequence tags (ESTs). BMC Genomics 2007, 8:134.

11. Liang C, Liu YS, Liu L, Davis AC, Shen YJ, Li QSQ: Expressed Sequence Tags With cDNA Termini: Previously Overlooked Resources for Gene Annotation and Transcriptome Exploration in Chlamydomonas reinhardtii. Genetics 2008, 179:83-93.
12. Liang C, Wang G, Liu L, Ji GL, Liu Y, Chen J, Webb JS, Reese G, Dean JF: WebTraceMiner: a web service for processing and mining EST sequence trace files. Nucleic Acids Res 2007, 35:W137-W142.

13. Chou HH, Holmes MH: DNA sequence quality trimming and vector removal. Bioinformatics 2001, 17:1093-1104.

14. Li S, Chou HH: Lucy 2: an interactive DNA sequence quality trimming and vector removal tool. Bioinformatics 2004, 20:2865-2866.

15. SeqClean:: ; [http://sourceforge.net/projects/seqclean].

16. Hillier L, Lennon G, Becker M, Bonaldo MF, Chiapelli B, Chissoe S, Dietrich N, DuBuque T, Favello A, Gish W, et al: Generation and analysis of 280,000 human expressed sequence tags. Genome Res 1996, 6:807-828

17. Peterson LA, Brown MR, Carlisle AJ, Kohn EC, Liotta LA, Emmert-Buck MR, Krizman DB: An improved method for construction of directionally cloned cDNA libraries from microdissected cells. Cancer Res 1998, 58:5326-5328.

18. Beißbarth T, Hyde L, Smyth GK, Job C, Boon WM, Tan SS, Scott HS, Speed TP: Statistical Modeling of Sequencing Errors in SAGE Libraries. Bioinformatics 2004, 20:i31-i39.

\section{doi:10.1186/1472-6750-12-16}

Cite this article as: Zhou et al:: Pattern analysis approach reveals restriction enzyme cutting abnormalities and other CDNA library construction artifacts using raw EST data. BMC Biotechnology 2012 12:16.

\section{Submit your next manuscript to BioMed Central and take full advantage of:}

- Convenient online submission

- Thorough peer review

- No space constraints or color figure charges

- Immediate publication on acceptance

- Inclusion in PubMed, CAS, Scopus and Google Scholar

- Research which is freely available for redistribution

Submit your manuscript at www.biomedcentral.com/submit
C Biomed Central 